\title{
Detection of Abandoned and Stolen Objects in Videos using Mixture of Gaussians
}

\author{
P. Sharon Femi \\ PG Scholar, \\ Department of Computer Science \& Engineering, \\ Sri Venkateswara College of Engineering,
}

\author{
K.Thaiyalnayaki \\ Associate Professor, \\ Department of Information Technology, \\ Sri Venkateswara College of Engineering,
}

\begin{abstract}
An efficient approach to detect abandoned and stolen object in live videos based on background subtraction and foreground analysis has been presented in this paper. The motivation is to produce alerts spontaneously in the presence of abandoned or stolen objects. To detect the abandoned and stolen objects, the focus is to determine the static regions that have recently changed in the scene by performing background subtraction. The most commonly used algorithm for background subtraction is the Gaussian Mixture Model (GMM). In the proposed detection method, an improved Multi- Gaussian Adaptive background model is employed for background subtraction. A simple split and merge method is used to detect the static region from which the static objects are identified. The time and presence of static objects, which may be either abandoned or stolen, are informed to the security officials by triggering an alarm. The method proposed in this research work is tested to run in real time and have shown expected results.
\end{abstract}

\section{Keywords}

Abandoned object, Background subtraction, static region, stolen object

\section{INTRODUCTION}

Over recent days, the need for video surveillance has grown tremendously in many areas to maintain social control, recognize and monitor threats, prevent, investigate criminal activity. In addition to security applications, video surveillance is also used to detect accidents in highways, military applications, left-luggage detection and parked vehicles detection in traffic monitoring [1],[2]. The system alerts the security officers of a burglary in progress or a suspicious individual loitering in a restricted area helping to avoid threat. Detection of objects plays an important role in surveillance system as the objects that are introduced in the foreground have to be detected instantaneously in time, in order to avoid dangerous situations. Earlier approaches reveal that identifying moving objects from a video sequence is a rudiment task for many computer-vision applications [3]. Hence, a common approach is to perform background subtraction, which detects the foreground objects from the portion of video frame that differs from the background model.

The system developed detects the objects placed idle for some time and alerts the security officials about the situation to take the necessary action for preventing any security threat. An abandoned object is a static object that is not in the scene before and stolen object is the object that was in the scene before but not present anymore. To detect the abandoned and stolen objects, the static objects should be determined first.
The static objects are the changes in the scene that remain in the same position for relatively long period of time.

The rest of this paper is organized as follows: Chapter 2 presents the related work. Chapter 3 presents the proposed work. Chapter 4 presents the background subtraction. Chapter 5 presents the static region and object detection method. Chapter 6 presents the experimental results in live videos.

\section{RELATED WORK}

Kong et al [3], detects non-flat abandoned objects by comparing a reference and target video sequences. The system uses GPS information to align the videos to find the frame pairs. It uses inter sequence geometric alignment to find suspicious areas, intra sequence geometric alignment to remove false alarms. The camera is mounted on a moving platform to scan along a specified trajectory for non-flat abandoned objects. The difficulty of the system is to cope with moving objects, presence of shadows, lighting conditions.

Stationary objects in multiple object tracking [4], detects the foreground objects with several moving objects and is inspired by human's visual cognition processes. This approach uses a specific processing layer corresponding to human attention for the detection. It also uses a Peripheral tracker and scene description layers to detect and maintain the static approach, based on object based detection and tracking in separate, parallel and interactive processes. It relies on tracking information to detect drop-off events. This system produced larger errors under bright lighting conditions.

Magno et al [5] employs an unobtrusive embedded platform. This method uses a wireless video sensor to detect the abandoned object. The system employs multimodal sensor integration which saves power consumption. The other specifications include PIR sensor, possibility to send alarm to a remote host wirelessly and low power consumption. The objective is to develop a multimodal video sensor with low power and low cost to detect abandoned objects. This uses new algorithms for energy efficient image processing without giving up the flexibility of in-field configuration. In spite of using a video sensor, the number of false positives is $13 \%$ of the total detected objects.

Singh et al [6] uses a dual-time background subtraction algorithm to dynamically update two sets of background. The dual background subtraction algorithm is based on Approximate Median Model. This method is dynamic, adaptive, non-probabilistic and intuitive in nature. It uses pixel color/ intensity information for background processing. The binary image is divided into a number of legitimate blobs. Once the blobs are generated, the system applies an algorithm for tracking of the abandoned objects. The system is robust to 
variations in lighting conditions and the number of people in the scene. However, the system mistakenly takes an immovable person as an abandoned object. The system does not classify stable objects as abandoned and removed objects

In robust detection of abandoned and removed objects in complex surveillance videos [7], the method detects abandoned and removed objects using GMM algorithm. The type of static regions is determined by a method that exploits context information. A matching method is used to detect the abandoned and removed object and it outperforms the edge based techniques. A person-detection process is integrated to differentiate static objects from stationary people. The system is robust to quick-lightning changes and occlusions. The accuracy of the detection is influenced by the size of the object, light conditions, and contrast situations

\section{PROPOSED SYSTEM}

In the present work, we provide a solution for detecting abandoned and stolen object in videos. Fig 1 shows the proposed system diagram. The system includes the following modules. i) Background subtraction ii) Static region detection iii) abandoned or stolen object alert detection.

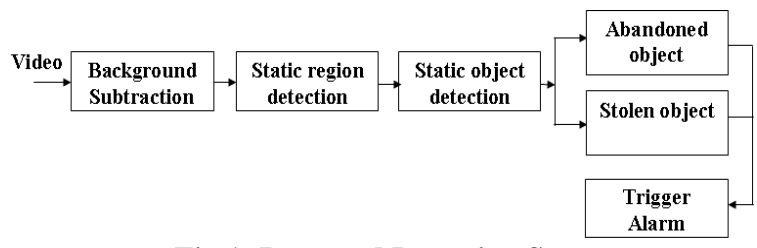

Fig 1: Proposed Detection System

The work introduced in this paper involves the following contributions.

i. Mixture of Gaussians are employed to detect the moving objects while subtracting the background.

ii. The frames are extracted from the live video and background subtracted video at the rate of 1 frame / 3 seconds.

iii. The extracted background subtracted frames are processed to determine the mismatched frames using a simple 'split and merge' algorithm.

iv. An alert is triggered on detection of abandoned or stolen object in videos.

\section{BACKGROUND SUBTRACTION}

In this section, we explain the background subtraction method employed to determine the static region. Background subtraction is performed using Mixture of Gaussians (MoG) method [7]. Mixture of Gaussians implements a classic multivariate Gaussian mixture model where every pixel is represented by a mixture of four Gaussian distributions. The modelling of the Gaussians is based on the Mahalanobis distance between the source and background model pixels. This model is designed to handle multimodal backgrounds with moving objects and illumination changes.

In Mixture of Gaussians, each pixel is characterized by its intensity in the RGB color model. The various steps involved in MoG are explained in the following subsections.

\subsection{Pixel characterization}

The probability of each pixel value $X_{t}$ is calculated as

$$
P\left(X_{t}\right)=\sum_{i=1}^{k} w_{i, t} * \eta\left(X_{t}, \mu_{i, t}, \Sigma_{i, t}\right)
$$

where,

$\mathrm{k}$ is the number of Gaussians (value may be $3-5$ )

$w_{i, t}$ is the weight associated to the Gaussian $\mathrm{i}$ at time $\mathrm{t}$

$X_{t}$ is the pixel value at time $\mathrm{t}$,

$\mu$ is the mean value of the $\mathrm{i}^{\text {th }}$ Gaussian distribution

$\Sigma$ is the covariance matrix

$\eta$ is the Gaussian probability density function defined as below:

$$
\eta\left(X_{t}, \mu, \Sigma\right)=\frac{1}{(2 \pi)^{n / 2}|\Sigma|^{1 / 2}} e^{-\frac{1}{2}\left(X_{t}-\mu_{t}\right)^{T} \Sigma^{-1}\left(X_{t}-\mu_{t}\right)}
$$

$n$ is the dimension of the intensity at the pixel X.

Each pixel has the same covariance matrix and is of the form $\Sigma_{i, t}=\sigma_{i, t}^{2} I$ and thus each pixel is characterized by a mixture of k Gaussians.

\subsection{Parameter Initialization}

The various parameters involved in Mixture of Gaussians are $\mathrm{k}, \Sigma, w_{i, t}$. In our system, $\mathrm{k}$ is set to $4, \Sigma$ is initialized to 50 and $w_{i, t}$ is initialized as in equation 3 .

$$
w_{i, t}=(1-\alpha) w_{i, t}+\alpha
$$

where $\alpha$ is the learning rate set to 0.001 . The mean and covariance matrix of the Gaussian at each pixel is continuously updated.

\subsection{Foreground Detection}

Initial foreground detection is made and the parameters are updated. Initial foreground detection is made by determining the ratio $r=w / \sigma$ and order the Gaussians following this ratio. The first $\mathrm{B}$ distributions are considered as the background model, where

$$
B=\arg \min _{b} \sum_{i=1}^{b} w_{i, t}>T
$$

This ensures that a high weight with a weak variance refers to a background pixel. The other distributions are considered to present a foreground distribution. The pixels at each frame are classified as foreground or background by calculating the Mahalanobis distance between the source and background model pixels, and comparing this distance to a threshold.

When a new frame enters at time $t+1$, a matching test is performed for every pixel. The Mahalanobis distance between the source and background model pixels are calculated using the formula,

$$
\text { Dist }=\operatorname{sqrt}\left(\left(X_{i+1}-\mu_{i, t}\right)^{T}-\sum_{i, t}^{-1}\left(X_{t+1}-\mu_{i, t}\right)\right)<k \sigma_{i, t}
$$

where,

$k$ and $T$ are the threshold set to 2.5 and 0.5 respectively.

Two cases may occur as a result of the matching test:

Case 1: Match found with one of the $\mathrm{k}$ Gaussians.

In this case, if the Gaussian identified is one among the B distributions, the pixel is classified as background, else it is foreground pixel.

Case 2: No match with any of the $\mathrm{k}$ Gaussians

In this case, the pixel is identified as foreground. To proceed for the next foreground detection, the parameters must be updated. 
Two cases occur in the foreground detection as below: Case a: A match found with one of the $\mathrm{k}$ Gaussians.

The updation of values for the matched component is as follows

$$
w_{i, t}=(1-\alpha) w_{i, t}+\alpha,
$$

where $\alpha$ is the constant learning rate

$\mu_{i, t+1}=(1-\rho) \mu_{i, t}+\rho X_{t+1}$

$\sigma_{i, t+1}^{2}=(1-\rho) \sigma_{i, t}^{2}+\rho\left(X_{t+1}-\mu_{i, t+1}\right) \cdot\left(X_{t+1}-\mu_{i, t+1}\right)^{T}$

Where

$$
\rho=\alpha \cdot \eta\left(X_{t}, \mu_{i, t}, \Sigma_{i, t}\right)
$$

For the unmatched component, the $\mu$ and $\Sigma$ remains unchanged and only the weight is updated as

$$
w_{j, t+1}=(1-\alpha) w_{j, t}
$$

Case b: No match with any of the k Gaussians

In this case, the distribution $\mathrm{k}$ is replaced with the parameters

$w_{k, t+1}=$ low prior weight

$\mu_{i, t+1}=X_{t+1}$

$\sigma_{k, t+1}^{2}=$ large initial variance

Once the parameters maintenance is made, foreground detection can be made and so on. The blind update employed by the method makes it less sensitive to initial conditions but tends to integrate stationary foreground objects into the background.

\section{STATIC REGION AND OBJECT DETECTION}

Static region is the region that has recently changed in the scene. To determine the static region from the background subtracted video, we propose an algorithm to determine the mismatched frames. Mismatched frames are the frames that contain a recent change in the scene and it may correspond to static object. The background subtracted video has to be converted to frames for the proper functioning of the algorithm. Thus the frames extracted from the background subtracted video consist of either black pixel of white pixel.

The algorithm works by processing $n$ number of frames simultaneously by parallel execution at any given time. The various steps involved in the algorithm are

i. Each frame is divided to sub-blocks of size, say $k$.

ii. The pixel count of each of the $k$ sub-block has to be determined

iii. If the number of white pixels of the sub-block is greater than a threshold value, that sub-block is considered as "white block", else the sub-block is considered as a "black block".

iv. The identified number of white blocks of a frame is stored in a buffer.

v. If the consecutive values of the buffer remain the same, then the corresponding frames are to be considered as mismatched frames.

vi. An alert is triggered to indicate the presence of abandoned or stolen object.
The process is repeated for the next $n$ frames of the background subtracted video till the end of the video so as to identify the mismatched frames.

\section{EXPERIMENTAL RESULTS}

The results of the experiment implemented for the proposed method, developed using Visual Studio, reveals that the system runs for live videos taken in the webcam to identify the static object. As live videos are monitored, instead of datasets, it is visualized that the system is able to detect the abandoned and stolen object with limited delay.

The importance is the system is able to identify the abandoned object if the lightening effects changes. Fig 2 shows some examples of the detected abandoned object in live videos. However, it is observed that the developed system suffers from shadow effects. The false negatives and false positives are avoided and the detection system developed is able to produce true positives for the live videos with limited delay.

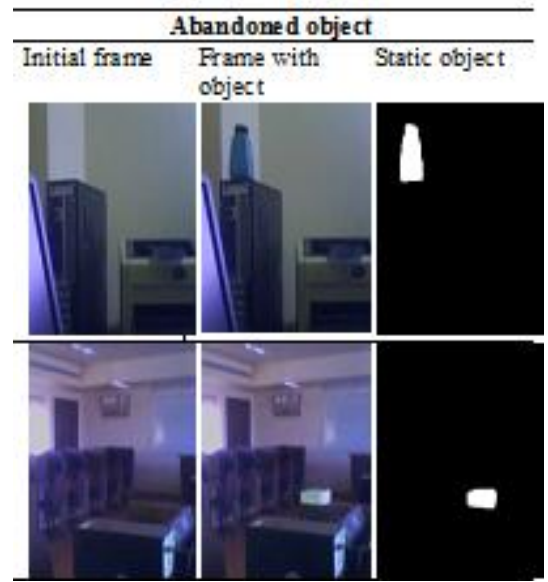

Fig 2: Examples of detected abandoned object

The system is evaluated with different set of objects of different size and types such as bottle, CD Drive, glue bottle, black board marker and the detection system is able to respond true positives for all the input live videos. The detection results for the abandoned and stolen object detection are presented in Table 1 and 2 .

Table 1. Detection results for abandoned object detection

\begin{tabular}{|c|c|c|}
\hline $\begin{array}{c}\text { Abandoned } \\
\text { object }\end{array}$ & $\begin{array}{c}\text { True } \\
\text { positives }\end{array}$ & $\begin{array}{c}\text { False } \\
\text { positive }\end{array}$ \\
\hline 5 & 5 & 0 \\
\hline
\end{tabular}

Table 2. Detection results for stolen object detection

\begin{tabular}{|c|c|c|}
\hline Stolen object & $\begin{array}{c}\text { True } \\
\text { positives }\end{array}$ & $\begin{array}{c}\text { False } \\
\text { positive }\end{array}$ \\
\hline 5 & 5 & 0 \\
\hline
\end{tabular}

The detection of some of the stolen objects is shown in Fig 3. 


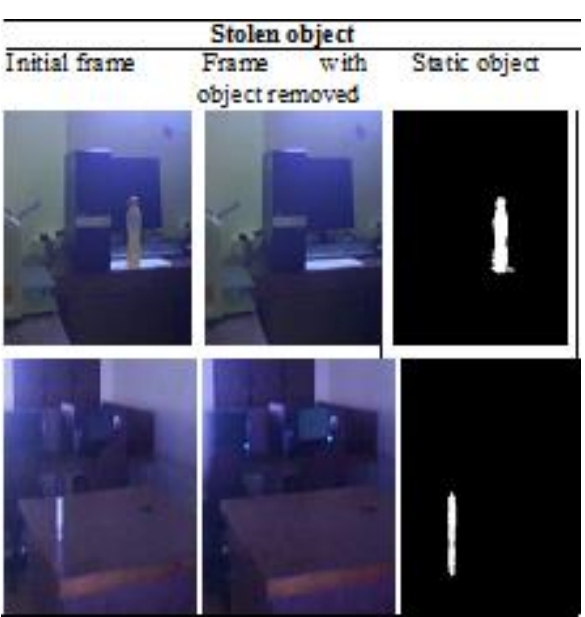

Fig 3: Examples of detected stolen object

\section{CONCLUSION}

A framework to detect the abandoned and stolen object in live videos has been implemented successfully in the proposed detection system. The mixture of Gaussians BGS method is used to detect both background and static foregrounds. The static region is determined using the simple split and merge algorithm. The static region is either classified as abandoned or stolen object and the information is given to the security officials instantaneously including the time of the presence of static object. The testing results, based on live videos, have proved that this approach can be effectively utilized for video surveillance applications.

\section{REFERENCES}

[1] E. Auvinet, E. Grossmann, C. Rougier, M. Dahmane, and J. Meunier 2006 Left-luggage detection using homographies and simple heuristics in PETS 2006, pp. $51-58$.

[2] N. Bird, S. Atev, N. Caramelli, R. Martin, O. Masoud, and N. Papanikolopoulos 2006 Real time, online detection of abandoned objects in public areas in Proc. IEEE Int. Conf. Robot. Automation, pp. 3775-3780.

[3] Hui Kong, Jean Ponce 2010 Detecting Abandoned Objects with a Moving Camera in Proc. IEEE Transactions on Image Processing,Vol 19,2010.

[4] S.Guler, J.A. Silverstein, and I.H.Pushee 2007 Stationary objects in multiple object tracking in Proc. IEEE Conf. Adv. Video Signal Based Surveillance, London, U.K.

[5] M.Magno, F.Tombari, D.Brunelli, L. Di Stefano and L.Benini 2009 Multimodal abandoned/removed object detection for low power video surveillance systems in Proc AVSBS09, pp 188-193.

[6] A. Singh, S. Sawan, M. Hanmadhu, V. K. Madasu, B. C. Lovell 2009 An abandoned object detection system based on dual background segmentation in Proc. IEEE Conf. Adv. Video and Signal Based Surveillance.

[7] Y Tian, Rogerio Schmidt Feris, Haowei Liu, A Hampapur and Ming-Ting Sun 2010 Robust Detection of Abandoned and Removed Objects in Complex Surveillance Videos in Proc. IEEE Transactions On Systems, Man, And Cybernetics-Part C: Applications And Reviews, Vol. 41. 